\title{
Exotic bottomonium-like hadrons
}

\author{
V. Baru \\ Helmholtz-Institut für Strahlen- und Kernphysik and Bethe Center for Theoretical Physics, \\ Universität Bonn, D-53115 Bonn, Germany \\ Institute for Theoretical and Experimental Physics, B. Cheremushkinskaya 25, 117218 Moscow, \\ Russia \\ P.N. Lebedev Physical Institute of the Russian Academy of Sciences, 119991, Leninskiy Prospect \\ 53, Moscow, Russia
}

\section{E. Epelbaum, A. A. Filin}

Ruhr University Bochum, Faculty of Physics and Astronomy, Institute for Theoretical Physics II, D-44780 Bochum, Germany

\section{Hanhart}

Forschungszentrum Jülich, Institute for Advanced Simulation, Institut für Kernphysik and Jülich Center for Hadron Physics, D-52425 Jülich, Germany

\section{A.V. Nefediev*}

P.N. Lebedev Physical Institute of the Russian Academy of Sciences, 119991, Leninskiy Prospect 53, Moscow, Russia

National Research Nuclear University MEPhI, 115409, Kashirskoe highway 31, Moscow, Russia E-mail: nefedievalebedev.ru

\section{Q. Wang}

Institute of Quantum Matter, South China Normal University, Guangzhou 510006, China

\begin{abstract}
In recent years, many hadronic states with heavy quarks have been observed which do not fit into the quark model scheme. Investigation of the nature and properties of such exotic states is an important task for the phenomenology of strong interactions. Most intriguing are charged states which at the same time contain a heavy quark-antiquark pair, since their minimal quark contents is four-quark and, therefore, their exotic nature is undoubted. A combined analysis of the existing experimental data on the production and decay channels of the isovector bottomonium-like states $Z_{b}(10610)$ and $Z_{b}(10650)$ (with the quantum number $J^{P C}=1^{+-}$) is performed in the framework of a coupled-channel approach which respects constraints from unitarity, analyticity and heavy quark spin symmetry. The latter symmetry, inter alia, allows one to relate properties of the $Z_{b}$ 's with those of their spin partners $W_{b J}$ (with the quantum numbers $J^{++}, J=0,1,2$ ) which differ from the $Z_{b}$ 's by the orientation of the heavy quark spins. As a result, the pole positions and the line shapes in different elastic and inelastic channels are predicted for the $W_{b J}$ 's in a parameter-free way. The developed approach admits a natural generalisation to other exotic states, in particular, to charmonia.
\end{abstract}

ALPS 2019 An Alpine LHC Physics Summit

April 22 - 27, 2019

Obergurgl, Austria

${ }^{*}$ Speaker. 


\section{Introduction}

In recent years, a lot of states in the spectrum of charmonium and bottomonium have been discovered experimentally. Some of them fit well into the quark model scheme while others do not. The latter states are called exotic hadrons. Most of them reside in the vicinity of strong thresholds which leave a strong footprint on their properties. Thus the discovery of the $X(3872)$ [1] — the first exotic charmonium-like state — started a new era in the heavy-quark spectroscopy with an important role played by the $B$-factories at $e^{+} e^{-}$colliders, such as Belle at KEK and BABAR at SLAC. A constant interest of the high energy physics community to the problem of exotic hadrons can be exemplified by a remarkable fact that the papers on the $X(3872)$ are the most cited publications of the Belle Collaboration since it was established.

Several approaches are claimed in the literature to be able to describe (at least some) properties of the exotic states with heavy quarks. Among those are

- the model of a compact tetraquark, that is a compact object made of the $(Q q)_{\overline{3}}$ and $(\bar{q} \bar{Q})_{3}$ pairs (here $Q$ and $q$ stand for the heavy and light quark, respectively, and the subscript indicates the colour state);

- the model of a hybrid meson - a compact object containing a quark-antiquark pair in the colour-octet state, $(Q \bar{Q})_{8}$, plus gluons;

- the hadro-quarkonium model which treats an exotic hadron as a colour-singlet heavy-quark core, $(Q \bar{Q})_{1}$, surrounded by a light-quark cloud;

- the model of a hadronic molecule — an extended object made of $(\bar{Q} q)_{1}$ and $(\bar{q} Q)_{1}$. Hadronic molecules are the central object of the present study.

Hadronic molecules are characterised by a large probability to find a two-hadron component in their wave function (see the review [2] and references therein). Any near-threshold resonance automatically becomes a strong candidate for a molecular state since the proximity of the strong threshold implies a large admixture of the hadron-hadron component in its wave function. Meanwhile, the nature of this state, the binding mechanisms in it and so on can vary from model to model. In the present work the molecular picture is employed to describe the properties of the charged $J^{P C}=1^{+-}$bottomonium-like states $Z_{b}(10610)$ and $Z_{b}(10650)$ observed by the Belle Collaboration in 2011 [3]. A molecular interpretation for these states was suggested in the pioneering work [4] and used in many later works, see, for example, Refs. [5, 6, 7, 8]. It should be noticed that one of the most important consequences of the presence of heavy quarks in a system is the possibility to relate its properties with those of other systems with the same quark contents but with a different orientation of the heavy-quark spins. The corresponding symmetry is known as the heavyquark spin symmetry (HQSS) and the states related by this symmetry are called the spin partners. The spin partners of the $Z_{b}$ 's are typically referred to as the $W_{b J}$ 's, with $J=0,1,2$ and the quantum numbers $J^{P C}=J^{++}[4,9,10]$. It has to be noticed that while the $Z_{b}$ 's are produced as intermediate states in pion decays from the $\Upsilon(10860)$ bottomonium, because of the opposite $C$-parity, their spin partners $W_{b J}$ 's can only be seen in the radiative decays of the $\Upsilon(10860)$ - see Fig. 1 . The latter observation implies that the probability of the corresponding decays are suppressed by the small 

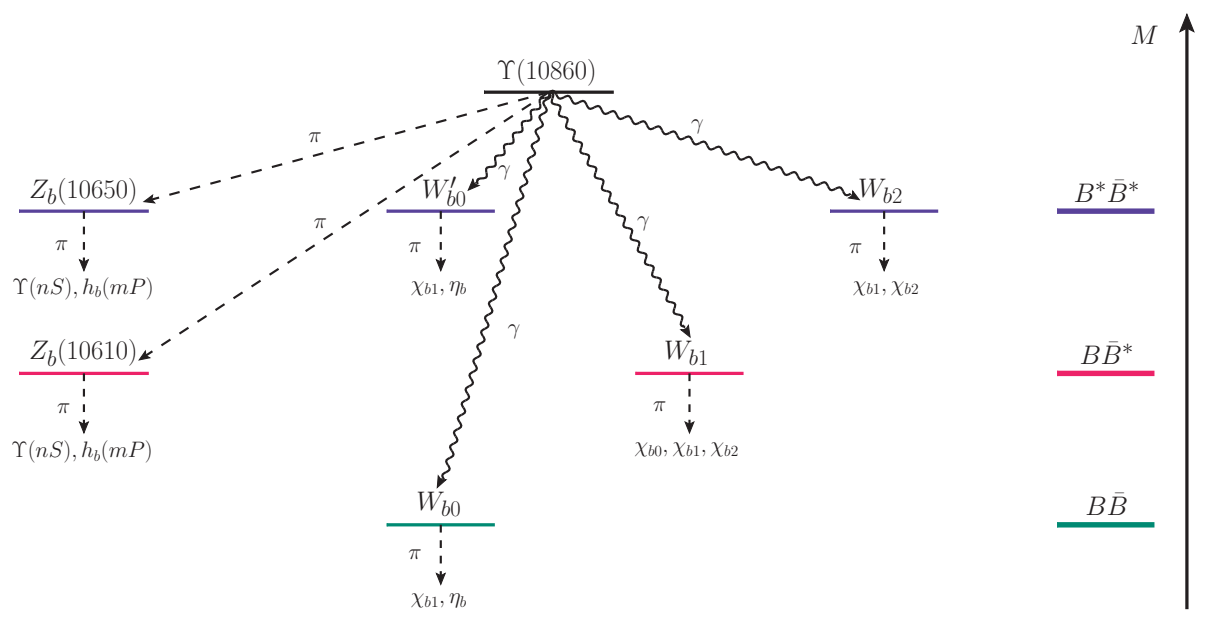

Figure 1: Schematic representation of the production and decay channels for the bottomonium-like states $Z_{b}(10610)$ and $Z_{b}(10650)$ and their spin partners $W_{b J}$ 's with $J=0,1,2$.

fine structure constant $\alpha=\frac{1}{137}$ compared to the pion decays. In the meantime, a huge statistics expected in future experiments, in particular, in the Belle-II one, should allow one to override this suppression.

\section{Common effective field theory for the $Z_{b}$ 's and $W_{b J}$ 's}

In the present work a common model-independent approach to the $Z_{b}$ 's and $W_{b J}$ 's is discussed using an effective field theory (EFT) framework $[8,12]$. The cornerstone of the approach is the interaction potential which respects HQSS and incorporates all relevant interactions up to the typical (soft) scale generated by the coupled-channel dynamics,

$$
p_{\text {typ }}=\sqrt{m_{B} \delta} \simeq 500 \mathrm{MeV}, \quad \delta=m_{B^{*}}-m_{B} \approx 45 \mathrm{MeV},
$$

where $m_{B}\left(m_{B^{*}}\right)$ is the $B^{(*)}$-meson mass so that two types of expansions are employed simultaneously: (i) in the HQSS violating parameter $\Lambda_{\mathrm{QCD}} / m_{b}$ (here $m_{b}$ is the $b$-quark mass) and (ii) in the ratio of the soft to hard scale, $p_{\text {typ }} / \Lambda$, where the hard scale $\Lambda \simeq 1 \mathrm{GeV}$. Then any physical quantity of interest ' $X$ ' calculated to the given order $v$ in a particular expansion can be represented as a series in powers of $\chi$ and the theoretical uncertainty comes from the neglected term of the order $v+1$,

$$
X^{(v)}(Q)=\sum_{n=0}^{v} \alpha_{n} \chi^{n}, \quad \delta X=X^{(v+1)}-X^{(v)}=\alpha_{v+1} \chi^{v+1} .
$$

Notice that the former expansion parameter $\left(\Lambda_{\mathrm{QCD}} / m_{b}\right)$ is fairly small, so that it is sufficient to work in the strict HQSS limit neglecting symmetry-violating corrections. In the meantime, given a large value of the soft scale quoted in Eq. (2.1), the latter parameter $\left(p_{\text {typ }} / \Lambda\right)$ appears to be of the order of $1 / 2$, so that the problem of the convergence of the EFT series has to be a special concern in the developed approach. Yet another consequence of the large value of the $p_{\text {typ }}$ is that the pionic dynamics has to be treated explicitly and, in particular, $D$ waves generated by this dynamics in 


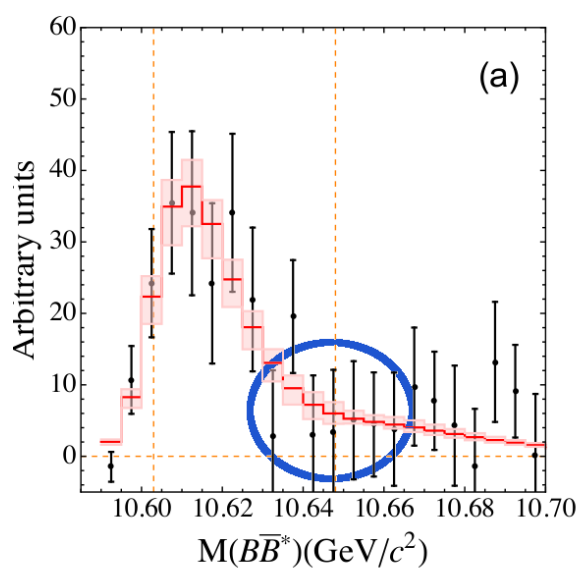

Figure 2: Line shape in the $B \bar{B}^{*}$ channel in the reaction $\Upsilon(10860) \rightarrow \pi B \bar{B}^{*}$. The experimental data [11] and the theoretical curve [8] with a $1 \sigma$ uncertainty are given by the black dots with error bars and by the red histogram with a band, respectively. The blue ellipse indicates the region around the $B^{*} \bar{B}^{*}$ threshold where a hump is naïvely expected due to the $B \bar{B}^{*}-B^{*} \bar{B}^{*}$ coupled-channels effects enhanced by the pion exchange.

the $B^{(*)} \bar{B}^{(*)}-\bar{B}^{(*)} B^{(*)}$ transitions are expected to play an important role and can not be neglected. However, the existing experimental data on the open-bottom decays $\Upsilon(10860) \rightarrow \pi B^{(*)} \bar{B}^{*}$ [11] do not support strong coupled-channel effects in the system $B \bar{B}^{*}-B^{*} \bar{B}^{*}$ (no signal near the $B^{*} \bar{B}^{*}$ threshold in the $B \bar{B}^{*}$ mass distribution - see Fig. 2) although the pion exchange would favour such transitions. A selfconsistent simultaneous description of the experimental data in various measured production and decay channels of the $Z_{b}$ 's ${ }^{1}[11,13]$,

$$
\Upsilon(10860) \rightarrow \pi B^{(*)} \bar{B}^{*}, \pi \pi h_{b}(m P)(m=1,2),
$$

can be achieved in an EFT approach if the system of coupled channels in the $1^{+-}$and $J^{++}(J=$ $0,1,2)$ channels includes all relevant partial waves,

$$
\begin{aligned}
& 1^{+-}: B \bar{B}^{*}\left({ }^{3} S_{1},-\right), B^{*} \bar{B}^{*}\left({ }^{3} S_{1}\right), B \bar{B}^{*}\left({ }^{3} D_{1},-\right), B^{*} \bar{B}^{*}\left({ }^{3} D_{1}\right) \\
& 0^{++}: B \bar{B}\left({ }^{1} S_{0}\right), B^{*} \bar{B}^{*}\left({ }^{1} S_{0}\right), B^{*} \bar{B}^{*}\left({ }^{5} D_{0}\right) \\
& 1^{++}: B \bar{B}^{*}\left({ }^{3} S_{1},+\right), B \bar{B}^{*}\left({ }^{3} D_{1},+\right), B^{*} \bar{B}^{*}\left({ }^{5} D_{1}\right) \\
& 2^{++}: B^{*} \bar{B}^{*}\left({ }^{5} S_{2}\right), B \bar{B}\left({ }^{1} D_{2}\right), B \bar{B}^{*}\left({ }^{3} D_{2}\right), B^{*} \bar{B}^{*}\left({ }^{1} D_{2}\right), B^{*} \bar{B}^{*}\left({ }^{5} D_{2}\right), B^{*} \bar{B}^{*}\left({ }^{5} G_{2}\right),
\end{aligned}
$$

and the terms up to the order $\mathscr{O}\left(p^{2}\right)$ are kept in the short-range interaction potential in the elastic (open-bottom) channels,

$$
V^{\mathrm{eff}}=V_{\mathrm{el}-\mathrm{el}}\left(\mathscr{O}\left(p^{2}\right)\right)+\sum_{\text {inel }} V_{\mathrm{el}-\text { inel-el }}+V_{\pi}
$$

in addition to the elastic-elastic transitions through the inelastic (hidden-bottom) channels and the one-pion exchange (OPE $)^{2}$. The corresponding Lippmann-Schwinger equation for the fully off-

\footnotetext{
${ }^{1}$ The total branching fractions in the channels $\Upsilon(10860) \rightarrow \pi \pi \Upsilon(n S)(n=1,2,3)$ were used in the combined fit to the data.

${ }^{2} \mathrm{Up}$ to the pion exchanges which are not given by iterations of the OPE (for example, a cross-box two-pion exchange) such a potential is NLO one. Since the neglected contributions are expected to be small, in what follows, the calculations with the potential (2.5) will be regarded as (almost) complete NLO.
} 

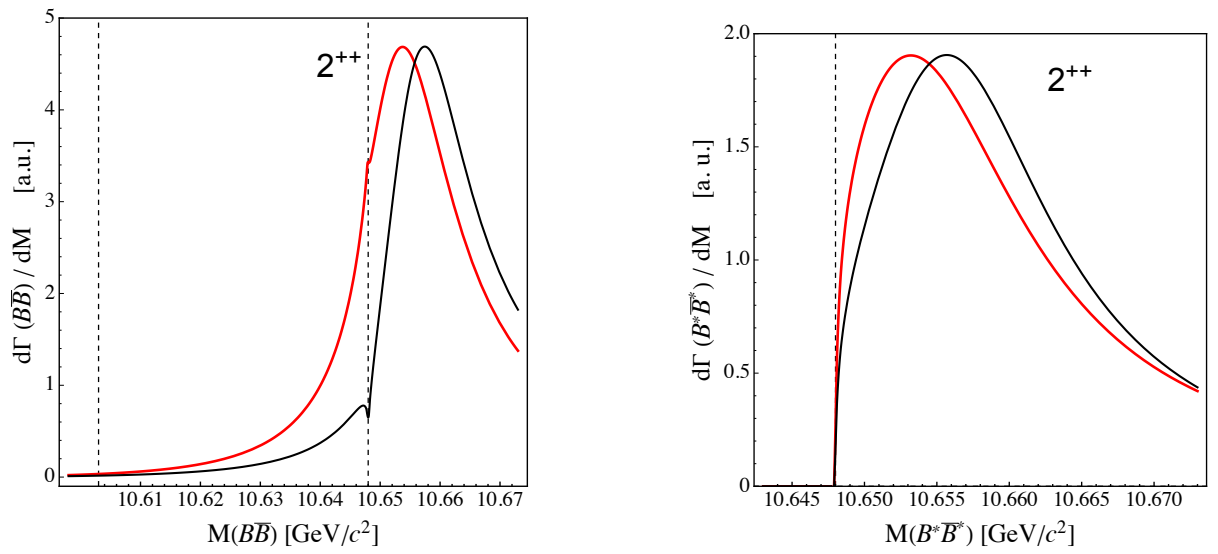

Figure 3: Predicted line shapes for $J^{P C}=2^{++}$in the elastic channels for the complete LO (red curve) and (almost) complete NLO (black curve).

shell elastic scattering amplitude $T$ is formulated as

$$
T_{\alpha \beta}\left(M, \boldsymbol{p}, \boldsymbol{p}^{\prime}\right)=V_{\alpha \beta}^{\mathrm{eff}}\left(\boldsymbol{p}, \boldsymbol{p}^{\prime}\right)-\sum_{\gamma} \int \frac{d^{3} q}{(2 \pi)^{3}} V_{\alpha \gamma}^{\mathrm{eff}}(\boldsymbol{p}, \boldsymbol{q}) G_{\gamma}(M, \boldsymbol{q}) T_{\gamma \beta}\left(M, \boldsymbol{q}, \boldsymbol{p}^{\prime}\right),
$$

where greek letters label elastic channels and $G_{\gamma}(M, \boldsymbol{q})$ is the Green's function in the $\gamma$-th elastic channel. It needs to be mentioned that renormalisability of the theory requires that the $S$-to- $D$ $\mathscr{O}\left(p^{2}\right)$ contact term is promoted from NLO to LO $[8,12]$. In particular, the overall description of the data appears to be nearly perfect $\left(\chi^{2} /\right.$ d.o.f $\left.=0.83\right)-$ see an example of the fitted line shape in Fig. 2; further examples can be found in Ref. [8]. Then the resulting EFT (i) provides a fairly good description of the data in all production and decay channels for the $Z_{b}$ 's; (ii) has all parameters (low-energy and coupling constants) fixed directly from the data; (iii) confirms that the data are compatible with HQSS; (iv) demonstrates a visible effect from the (long range) pion exchange; (v) describes the data near the $B^{*} \bar{B}^{*}$ threshold in the $B \bar{B}^{*}$ mass distribution, as shown in Fig. 2, that is the transitions mediated by the pion exchange are tamed in this energy region by the short-range interactions. The EFT just built can be used to make parameter-free predictions for the properties of the spin partner states $W_{b J}$ with $J=0,1,2$. The predicted line shapes, pole positions and branching fractions can be found in Ref. [12]. In particular, all $W_{b J}$ 's (as well as both $Z_{b}$ 's) appear to be above-threshold resonances (to be confronted with the pionless theory which predicts them all to be virtual states). As explained in Eq. (2.2) above, the theoretical uncertainty of the chiral expansion can be estimated by comparing LO with NLO as

$$
\delta E \simeq E_{\text {typ }} \frac{p_{\text {typ }}}{\Lambda} \simeq 15 \frac{500}{1000} \simeq 7.5 \mathrm{MeV},
$$

where the difference between the LO and NLO calculations is exemplified in Fig. 3 and the typical energy $E_{\mathrm{typ}}$ is estimated from the position of the peak in the line shape.

\section{Conclusions}

We conclude that the theoretical EFT approach based on the molecular picture for the $Z_{b}$ 's (i) 
is compatible with constraints from unitarity, analyticity, and HQSS; (ii) incorporates all relevant types of interactions and scales; (iii) is able to explain the existing data on the $Z_{b}(10610)$ and $Z_{b}(10650)$; (iv) is suitable to predict in a parameter-free way the spin partners $W_{b J}$ 's.

Further theoretical developments of this approach should include (i) a complete NLO calculation to improve the theoretical accuracy; (ii) inclusion of the pion final-state interaction to allow for the parameters extraction from the relevant data, for example, for the decays $\Upsilon(10860) \rightarrow \pi \pi \Upsilon(n S)$ ( $n=1,2,3)$; (iii) inclusion of the compact components of the wave function to allow one to treat isoscalar molecules; (iv) extension to the $S U$ (3) flavour group for light quarks to accomodate for the molecular states with strange quarks; (v) further tests of the accuracy of HQSS (that is especially relevant in the $c$-sector) to better control the theoretical uncertainties. This work is under way and the results will be reported elsewhere.

Partial support from the DFG (Grant No. TRR110) and the NSFC (Grant No. 11621131001) through the funds provided to the Sino-German CRC 110 "Symmetries and the Emergence of Structure in QCD" and the Russian Science Foundation (grant 18-12-00226) is gratefully acknowledged.

\section{References}

[1] S. K. Choi et al. [Belle Collaboration], Observation of a narrow charmonium-like state in exclusive $B^{ \pm} \rightarrow K^{ \pm} \pi^{+} \pi^{-} J / \psi$ decays, Phys. Rev. Lett. 91 (2003) 262001.

[2] F. K. Guo et al., Hadronic molecules, Rev. Mod. Phys. 90 (2018) 015004.

[3] I. Adachi [Belle Collaboration], Observation of two charged bottomonium-like resonances, arXiv:1105.4583 [hep-ex].

[4] A. E. Bondar et al., Heavy quark spin structure in $Z_{b}$ resonances, Phys. Rev. D 84 (2011) 054010.

[5] T. Mehen and J. Powell, Line shapes in $\Upsilon(5 S) \rightarrow B^{(*)} \bar{B}^{(*)} \pi$ with $Z(10610)$ and $Z(10650)$ using effective field theory, Phys. Rev. D 88 (2013) 034017.

[6] C. Hanhart et al., Practical Parametrization for Line Shapes of Near-Threshold States, Phys. Rev. Lett. 115 (2015) 202001.

[7] F.-K. Guo et al., Interplay of quark and meson degrees of freedom in near-threshold states: A practical parametrization for line shapes, Phys. Rev. D 93 (2016) 074031.

[8] Q. Wang et al., Line shapes of the $Z_{b}(10610)$ and $Z_{b}(10650)$ in the elastic and inelastic channels revisited, Phys. Rev. D 98 (2018) 074023.

[9] M. B. Voloshin, Radiative transitions from Upsilon(5S) to molecular bottomonium, Phys. Rev. D 84 (2011) 031502.

[10] T. Mehen and J. W. Powell, Heavy Quark Symmetry Predictions for Weakly Bound B-Meson Molecules, Phys. Rev. D 84 (2011) 114013.

[11] A. Garmash et al. [Belle Collaboration], Observation of $Z_{b}(10610)$ and $Z_{b}(10650)$ Decaying to $B$ Mesons, Phys. Rev. Lett. 116 (2016) 212001.

[12] V. Baru et al., Spin partners $W_{b J}$ from the line shapes of the $Z_{b}(10610)$ and $Z_{b}(10650)$, Phys. Rev. D 99 (2019) 094013.

[13] A. Bondar et al. [Belle Collaboration], Observation of two charged bottomonium-like resonances in $\Upsilon(5 S)$ decays, Phys. Rev. Lett. 108 (2012) 122001. 